\title{
Explicit Solutions of the Wave Equation on Three Dimensional Space-Times: Two Examples with Dirichlet Boundary Conditions on a Disk
}

\author{
Daniel Boykis and Patrick Moylan \\ Physics Department \\ The Pennsylvania State University \\ Abington College \\ Abington, Pennsylvania 19001 USA
}

Received: January 10, 2006

Accepted: February 20, 2006

\begin{abstract}
We study solutions of the wave equation with circular Dirichlet boundary conditions on a flat twodimensional Euclidean space, and we also study the analogous problem on a certain curved space which is a Lorentzian variant of the 3-sphere. The curved space goes over into the usual flat space-time as the radius $R$ of the curved space goes to infinity. We show, at least in some cases, that solutions of certain Dirichlet boundary value problems are obtained much more simply in the curved space than in the flat space. Since the flat space is the limit $R \rightarrow \infty$ of the curved space, this gives an alternative method of obtaining solutions of a corresponding problem in Euclidean space.
\end{abstract}

\section{INTRODUCTION}

Solutions of the wave equation with appropriate circular or cylindrical boundary conditions have many practical applications in engineering and physics. The paradigm of such textbook problems is that describing vibrations of a circular membrane (the shape of a drum) requiring solutions of the wave equation in a 2+1 dimensional Minkowski space. These solutions must vanish on the circular boundary of the membrane and thus lead to the zeros of the Bessel functions [1]. Similarly, cylindrical wave guides in threespace provide another important example. A theoretical application of much current interest, requiring such solutions, is the computation of Casimir energies for cylindrical and spherical boundary conditions.

We show here that it is just as easy to set up such problems in a certain curved space of which the flat Minkowski space is a limiting case. The curved space which we consider in section III is a Lorentzian variant of the 3-sphere. In general relativity it is just the Einstein universe, only in one lower spatial dimension, since here we deal with $2+1$ dimensions instead of the $3+1$ (i.e., four) dimensional space-time of physical reality. The relation between Minkowski space and the Einstein universe is analogous to that of a stereographic projection of the 3-sphere onto a tangent plane of one of its points. Owing to the indefiniteness of the scalar product in Minkowski space, the formulas of stereographic projection are more complicated that the usual formulas of stereographic projection, since it is a projection onto Minkowski space instead of onto Euclidean space.

It is clear as the radius $R$ of the curved space goes to infinity that solutions of the wave equation in the curved space must go over into corresponding solutions of 
the wave equation in flat space-at least locally. Our main reasons for looking at such problems in curved space is 1) the greater regularity and convergence properties afforded by working with fields over a compact space [2]; and 2) contrary to what one might expect, some problems formulated in the curved space may have considerably simpler solutions than those in flat space. Indeed, one of the authors [PM] has shown this to be the case for the calculation of the Casimir energy, at least for the lowest dimensional case [3].

\section{WAVE EQUATION FOR A MASSLESS SCALAR FIELD ON A 3D MINKOWSKI SPACE}

Let $M_{0}$ denote three dimensional Minkowski space, which is $\mathfrak{R}^{3}$ as a vector space, but whose metrical structure is that of a Lorentzian manifold with infinitesimal arc length in an inertial coordinate system given by

$$
d x^{2}=d x_{0}^{2}-d x_{1}^{2}-d x_{2}^{2} .
$$

We will be concerned with solutions of the conformally invariant wave equation, which in Cartesian coordinates on $M_{0}$ is written as

$$
\left(\frac{1}{c^{2}} \frac{\partial^{2}}{\partial x_{0}^{2}}-\frac{\partial^{2}}{\partial x_{1}^{2}}-\frac{\partial^{2}}{\partial x_{2}^{2}}\right) \psi(x)=0,
$$

where $\psi(x)\left[\equiv \psi\left(x_{0}, x_{1}, x_{2}\right)\right]$ is a suitably differentiable function on $M_{0}$ and $c$ is the speed of light in a vacuum.

Another set of coordinates on $M_{0}$ is $(\mathrm{t}, \mathrm{r}, \varphi)$ with $\mathrm{x}_{0}=\mathrm{t}, \mathrm{x}_{1}=\mathrm{r} \cdot \cos (\varphi)$, and $\mathrm{x}_{2}=$ $r \cdot \sin (\varphi)$, where $-\infty<t<\infty, 0 \leq r \leq \pi$, and 0 $\leq \varphi<2 \pi$. In the coordinates the wave equation becomes

$$
\Delta \psi-\frac{1}{c^{2}} \frac{\partial^{2} \psi}{\partial t^{2}}=0
$$

where

$$
\Delta \equiv \frac{\partial^{2}}{\partial r^{2}}+\frac{1}{r} \frac{\partial}{\partial r}+\frac{1}{r^{2}} \frac{\partial^{2}}{\partial \varphi^{2}}
$$

is the familiar Laplacian on $\mathfrak{R}^{2}$.

Solutions of equation (3) which are regular at the origin are:

$$
\psi_{k m}^{ \pm}(t, r, \varphi)=J_{m}(k r) e^{\imath m \varphi} e^{ \pm \imath \omega t}
$$

with $0<k<\infty$ and $m=0, \pm 1, \pm 2, \pm 3 \ldots$, and with $\omega=k c \in \mathfrak{R}$. A standard method of obtaining solutions (5) of equation (3) is that of separation of variables [4]. For completeness we present in the Appendix a detailed derivation of (5) using this method.

Dirichlet boundary conditions on the circle of radius $a$, which we consider here, means that for each Bessel function of order $n$ we require

$$
J_{n}(k a)=0 .
$$

This means $k a$ is a root of this equation. Since the positive roots of equation (5) are countable in number, we index them as $k a=$ $k_{n, j} a$, with $\mathrm{j}=1,2,3 \ldots$ (For each $\mu \in \mathfrak{R}$ we have a countable number of real roots of the equation $\left.J_{\mu}(k a)=0[1]\right)$.

It is easily verified that a general solution of equation (3) can be written as

$$
\psi(t, r, \varphi)=\cos (c \sqrt{-\Delta} t) f(r, \varphi)+\frac{1}{\sqrt{-\Delta}} \sin (c \sqrt{-\Delta} t) g(r, \varphi)
$$

where

$$
f(r, \varphi)=\psi(0, r, \varphi)
$$

and

$$
g(r, \varphi)=\left.\frac{\partial \psi}{\partial t}(t, r, \varphi)\right|_{t=0}
$$

are the initial conditions which describe the field $\psi(t, r, \varphi)$ at time $t=0$. In fact, it is proved in reference [1] (vol. 2, p 160) that equation (7) is the most general solution of equation (3). Since we have imposed the Dirichlet boundary conditions we must insist that 


$$
f(a, \varphi)=0 \text { and } g(a, \varphi)=0 .
$$

Due to the completeness of the basic solutions, equations (5), ${ }^{\text {a }}$ we can expand $f$ and $g$ in terms of these basic solutions as

$$
f(r, \varphi)=\sum_{m, j=0}^{\infty} a_{m, j} J_{m}\left(k_{m, j} r\right) e^{\imath m \varphi}
$$

and

$$
g(r, \varphi)=\sum_{m, j=0}^{\infty} b_{m, j} J_{m}\left(k_{m, j} r\right) e^{\imath m \varphi}
$$

where $k_{m, j}$ a is a positive root of equation (5). Through the inversion formulas of these equations the coefficients $a_{m, j}$ and $b_{m, j}$ may explicitly be given in terms of the initial functions $f(r, \varphi), \quad g(r, \varphi)$ and the $J_{m}\left(k_{m, j} r\right) e^{\imath m \varphi}$ [8].

Very often for a particular problem it is only necessary to know the series, equations (11) and (12), to some finite number of terms. Thus, in such cases we are interested in only the first $N$ positive zeros of the Bessel functions $J_{n}(x)$ of integer order $n$. Computational routines to find these zeros, using software such as Maple, are easy. We can do the job in Maple 9.5 with the following two simple lines of code:

$g:=(i) \rightarrow$ fsolve $\left(\right.$ BesselJ $\left.(0, x)=0, x(i-1)^{*} 2^{*} P i . . i^{*} 2^{*} P i\right)$; seq(g(i),i=1..10);

This routine has the advantage that it can easily be adapted to the Gegenbauer case in the next section. Of course, with Maple 9.5 one may also use the Maple routine "BesselJZeros $\left(n, v_{1} . . v_{2}\right)$ " which gives $v_{2}-v_{1}$ consecutive zeros of the Bessel function of order $\mathrm{n}$ starting with the $\mathrm{v}_{1}$ 'st zero.

III. WAVE EQUATION FOR A MASSLESS SCALAR FIELD ON A LORENTZIAN VARIANT OF THE 3SPHERE

Next consider the space-time $M$, a three dimensional version of a compactification of the Einstein universe, namely,

$$
M=\left\{\left(u_{0}, u_{1}, u_{2}, u_{3}, u_{4}\right) \mid u_{0}^{2}+u_{4}^{2}=u_{1}^{2}+u_{2}^{2}+u_{3}^{2}=R^{2}\right\}
$$

Clearly $M \cong S^{1} \times S^{2}$. We may introduce spherical coordinates on $M$ as follows:

$$
\begin{gathered}
u_{4}=R \cos (\tau), u_{0}=R \sin (\tau) \\
u_{1}=R \sin (\vartheta) \cos (\varphi), u_{2}=R \sin (\vartheta) \sin (\varphi) \\
u_{3}=R \cos (\vartheta)
\end{gathered}
$$

\footnotetext{
a By completeness we mean completeness in a certain Hilbert space $\mathscr{H}$ of solutions of the zero mass wave equation, equation (3), see reference [5]. This remark also makes the meaning of ($\Delta)^{1 / 2}$ precise: it is a fact that $-\Delta$ has a self-adjoint closure in $\mathscr{\Psi}[6]$, and thus we take $(-\Delta)^{1 / 2}$ to be the unique square root of the self-adjoint operator $-\Delta[7]$.

${ }^{\mathrm{b}}$ Many such additional routines can be found, for example, on the "Maple User Group" site: http://www-math.rwth-aachen.de/MapleAnswers/ 155.html. We note that some of the routines given at this site-which are written for Maple 4 or 5-do not work for Maple 9.5 without modification.
}

with ranges of the angular parameters being $0 \leq \tau<2 \pi, 0 \leq \vartheta<\pi, 0 \leq \varphi<2 \pi$. An $\mathrm{SO}(2) \times \mathrm{SO}(3)$ invariant metric on $M$ is

$$
d \sigma^{2}=R^{2}\left(d \tau^{2}-d \rho^{2}\right)
$$

where $d \tau$ and $d \rho$ denote infinitesimal arc length on the unit sphere $S^{1}$ in $\Re^{2}$ and $S^{2}$ in $\mathfrak{R}^{3}$, respectively.

$\mathrm{SO}(2,3)$ acts as the group of conformal transformations $M$ [9], and there is a conformal imbedding $\pi^{-1}$ of the Minkowski space $M_{0}$ into $M$, given by $\pi^{-1}(x)=u$, with the inverse mapping given by a Lorentzian variant of stereographic projection. Specifically we have

$$
u_{-1}=R p F, u_{i}=R p x_{i}, u_{3}=R p D
$$

where $i=0,1,2$ and 


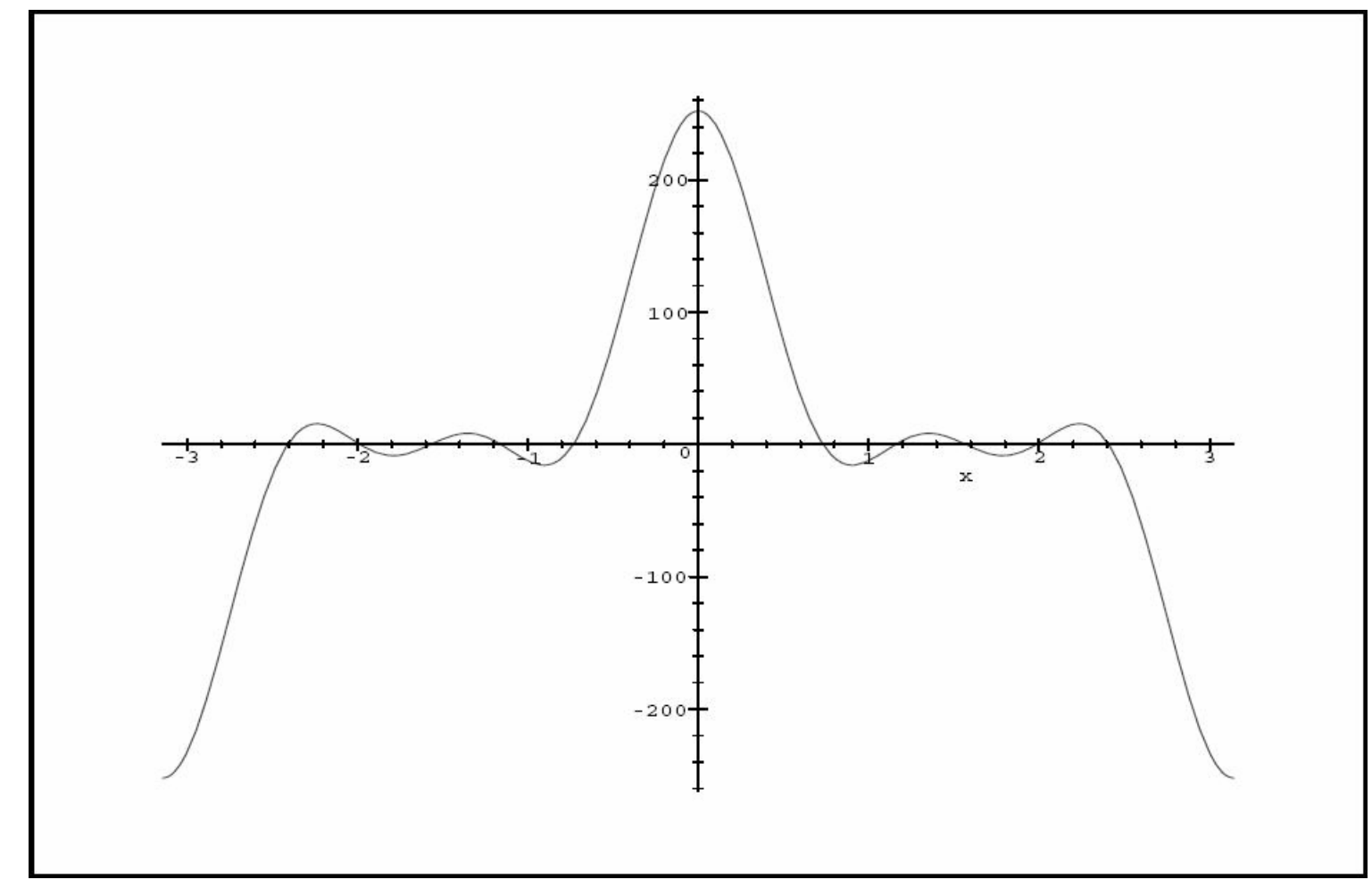

Figure 1. Graph of $C_{5}^{3}(\cos (\vartheta))$ for $-\pi \leq \vartheta \leq \pi$.

$$
p=\left\{F^{2}+\frac{c^{2} x_{0}^{2}}{R^{2}}\right\}^{-1 / 2}, \quad F=1-\frac{x^{2}}{4 R^{2}}, \quad D=1+\frac{x^{2}}{4 R^{2}}
$$

where $c^{2} x^{2}=x_{0}^{2}-c^{2} x_{1}^{2}-c^{2} x_{2}^{2}$.

Now $\pi^{-1}$ is conformal, since

$$
d \sigma^{2}=p^{2} d x^{2}
$$

Note that equations (14) and (16) give [9]

$$
\begin{gathered}
c x_{0}=\frac{2 R \sin (\tau)}{(\cos (\tau)+\cos (\vartheta))} \\
x_{1}=\frac{2 R \sin (\vartheta) \cos (\varphi)}{(\cos (\tau)+\cos (\vartheta))} \\
x_{2}=\frac{2 R \sin (\vartheta) \sin (\varphi)}{(\cos (\tau)+\cos (\vartheta))}
\end{gathered}
$$

The conformally invariant wave equation on $M$ is [10]

$$
\frac{\partial^{2}}{\partial r^{2}} \psi-\Delta_{S^{2}} \psi=\frac{1}{4 R^{2}} \psi
$$

Where $\Delta_{S^{2}}$ is the Laplacian on $S^{2}$ which in spherical coordinates is

$$
\Delta_{S^{2}}=-\frac{1}{\sin (\vartheta)}\left(\frac{\partial}{\partial \vartheta} \sin (\vartheta) \frac{\partial}{\partial \vartheta}+\frac{1}{\sin (\vartheta)} \frac{\partial^{2}}{\partial \varphi^{2}}\right)
$$

Solutions of the wave equation (compare to the 1+3 dimensional case in [11]) are: 


$$
\begin{gathered}
\psi_{E \ell m}(\tau, \vartheta, \varphi)=\left[\frac{(2 \ell+1)(\ell-m) !}{(\ell+m) !}\right]^{1 / 2} e^{i E \tau} P_{\ell}^{m}(\cos (\vartheta)) \\
=\frac{(-1)^{m}(2 m) !}{m ! 2^{m}}\left[\frac{(2 \ell+1)(\ell-m) !}{(\ell+m) !}\right]^{1 / 2} e^{\imath E \tau} \sin ^{m}(\vartheta) C_{\ell-m}^{m+1 / 2}(\cos (\vartheta)) e^{\imath m \varphi}
\end{gathered}
$$

with $\ell=1,2,3 \ldots ; 0 \leq m \leq \ell$, with $m \in \mathcal{Z}^{+}$. The $C_{\ell-m}^{m+1 / 2}(\cos (\vartheta))$ are Gegenbauer polynomials [12] and the $P_{\ell}^{m}(\cos (\vartheta))$ are associated Legendre functions [12]. The $\psi_{E \ell m}(\tau, \vartheta, \varphi)$ are normalized up to an inessential constant. Substituting these solutions into the wave equation gives the following spectral equation:

$$
E^{2}-\frac{1}{R^{2}} \ell(\ell+1)=\frac{1}{4 R^{2}} .
$$

Now for reasons made clear in the introduction we wish to consider the analog of equation (6) for the case at hand, i.e. Dirichlet boundary conditions on the region whose boundary is the great circle on $S^{2}$ which is contained in a plane parallel to the $x_{1}-x_{2}$ plane and is specified by the equation $\vartheta=\pi / N$. This means

$$
C_{\ell+m-1}^{-m+1 / 2}(\cos (\pi / N))=0 .
$$

which leads us to the question of zeros of Gegenbauer polynomials. (If we let $q=e^{2 \pi \imath \vartheta}$ then we see that the condition $\vartheta=\pi / N$ means $q^{2 N}=1$, i.e. $q^{1 / 2}$ is an Nth root of unity. We can easily adapt the Maple code of section II to write a program in Maple 9.5 for determining the zeros of the Gegenbauer polynomials:

$$
\begin{aligned}
& h:=(I, N, a, b) \rightarrow \text { fsolve }\left(\text { GegenbauerC }(a, b, \cos (x))=0, x,(i-1)^{*} 2^{*} \mathrm{Pi} / N . .{ }^{*} 2^{*} \mathrm{Pi} / N\right) ; \\
& S:=(N, a, b) \rightarrow \operatorname{seq}(h(I, N, a, b), i . . N) ;
\end{aligned}
$$

We can get all of the roots of a particular $C_{a}^{b}(\cos (\vartheta))$ by choosing $N$ sufficiently large. If we take the case of $C_{5}^{3}(\cos (\vartheta))$ for example, we find that $N=12$ is sufficient, and computing $S(12,5,3)$ we get the following roots in the interval $[0,2 \pi]$ (after cleaning up the output slightly):

$$
\begin{aligned}
& \text { 0.7325417151, 1.157199971, } \\
& \text { 1.984392683, 2.409050938, } \\
& \text { 3.874134369, 4.298792624, } \\
& \text { 5.127985336, 5.550643592 }
\end{aligned}
$$

Using the with(plots) package of Maple we may easily plot the graph of $C_{5}^{3}(\cos (\vartheta))$ in the range $[-\pi, \pi]$ to obtain agreement of the above list of zeros with the plot. (Due to the $\cos (\theta)$ factor $C_{a}^{b}(\cos (\vartheta))$ is periodic with period $2 \pi$.) Figure 1 shows the plot of this function.

Actually what we have just given is not quite what we need for our problem, which is to find all Gegenbauer polynomials which vanish at a particular value of the independent variable. However, one should be able to adapt the just described routine to the problem of finding out which Gegenbauer polynomials $C_{a}^{b}(\cos (\vartheta))$ have zeros at a particular value of $\mathrm{x}$, or we can use the following Maple routine to determine those Gegenbauer polynomials which have zeros at a particular value of $x$ :

$$
\begin{aligned}
& \mathrm{k}:=(\mathrm{I}, \mathrm{N} . \mathrm{m}, \theta) \rightarrow \mathrm{fsolve}(\text { GegenbauerC }(\mathrm{a}-\mathrm{m}, \mathrm{m}+1 / 2, \cos (\theta)))=0, \mathrm{a},(\mathrm{i}-1.1 . . \mathrm{i}+.1) \\
& \mathrm{T}:=(\mathrm{N}, \mathrm{m}, \theta) \rightarrow \operatorname{seq}(\mathrm{k}(\mathrm{l}, \mathrm{N}, \mathrm{m}, \theta), \mathrm{i}=1 . . \mathrm{N})
\end{aligned}
$$


It is possible to use this maple routine to convince oneself, in a case by case evaluation, of the validity of the following proposition:

Proposition: for $v>0, \mathrm{k}>0$

$$
C_{k}^{v}(\cos (\pi / 2))=0 \quad \Leftrightarrow \quad \mathrm{k} \text { is odd. }
$$

For example, the above routine for $T(10,1, \theta=\pi / 2)$ gives all integer values less than 10 of the index $a$ of the Gegenbauer polynomials $C_{a-1}^{b}(\cos (\vartheta))$ for which $C_{a-1}^{b}(\cos (\pi / 2))=0$. They are: $a=2,4$,
6,8 . This verifies the proposition, since $\mathrm{k}=$ $a-1=1,3,5,7$ which are all odd integers. This proposition is easily established in the case of integer $v$ by using the defining properties of the associated Legendre polynomials with positive integer values $v$ : one convinces oneself that for an odd positive integer $v$ the polynomial contains an overall factor $\cos (\theta)$ which is zero for $\theta=$ $\pi / 2$.

We now wish to use this result to write down, in analogy to equation (7), the general solution to equation (20) with Dirichlet boundary conditions on the equator of a sphere $[13,14]$. We have:

$\psi(\tau, \vartheta, \varphi)=\cos \left(\sqrt{-\Delta_{s}^{2}+\frac{1}{4 R^{2}}} \tau\right) f(\vartheta, \varphi)+\left(\sqrt{-\Delta_{s}^{2}+\frac{1}{4 R^{2}}}\right)^{-1} \sin \left(\sqrt{-\Delta_{s}^{2}+\frac{1}{4 R^{2}}} \tau\right) g(\vartheta, \varphi)$

where

$$
f(\vartheta, \varphi)=\psi(t, \vartheta, \varphi)
$$

and

$$
g(\vartheta, \varphi)=\left.\frac{\partial \psi(t, \vartheta, \varphi)}{\partial t}\right|_{t=0}
$$

are the initial conditions which describe the field $\psi(t, \vartheta, \varphi)$ at time $t=0$. imply that

The Dirichlet boundary conditions

$$
f(\pi / 2, \varphi)=0 \text { and } g(\pi / 2, \varphi)=0 .
$$

As in the previous section we can expand $f$ and $g$ in terms of the basic solutions in equation (22) as:

$$
f(\vartheta, \varphi)=\sum_{\ell=0}^{\infty} \sum_{\substack{-\ell<m<\ell \\ \ell-m \text { odd }}} c_{\ell, m} \sin ^{m}(\vartheta) C_{\ell-m}^{m+1 / 2}(\cos (\vartheta)) e^{\imath m \varphi}
$$

and

$$
g(\vartheta, \varphi)=\sum_{\ell=0}^{\infty} \sum_{\substack{\ell<m<\ell \\ \ell-m \text { odd }}} d_{\ell, m} E_{\ell} \sin ^{m}(\vartheta) C_{\ell-m}^{m+1 / 2}(\cos (\vartheta)) e^{\ell m \varphi}
$$

The coefficients $c_{m, j}$ and $d_{m, j}$ may explicitly be given in terms of the initial functions $f(r, \varphi)$ and $g(r, \varphi)$ and the $C_{\ell-m+1 / 2}^{m+1 / 2}(\cos (\vartheta)) e^{\imath m \varphi}$ by the inversion formulas, which can be obtained by adapting methods in [14] to the case at hand.

It is remarkable at least for this special case that we may write down a complete solution to the problem in curved space, whereas for the analogous problem in flat space, much more work is necessary. In the flat space case we require specific knowledge of the behavior of zeros Bessel functions for large values of the argument, since the equator of the sphere corresponds under stereographic projection to a circle of radius $2 R$ in flat space, and the flat space is obtained as the limit $R \rightarrow \infty$ of the curved space case. 


\section{APPENDIX}

In this appendix we construct solutions of the wave equation in polar coordinates:

$$
\frac{\partial^{2} \psi}{\partial t^{2}}-c^{2}\left[\frac{\partial^{2} \psi}{\partial r^{2}}+\frac{\partial \psi}{\partial r}+\frac{1}{r^{2}}\right]=0 \text {. }
$$

In this Appendix (only) we use, as is customary, $\vartheta$ to denote the angle of rotation in the plane. In the above we used $\varphi$ for the azimuthal angle instead of $\vartheta$ so as not to confuse it with the polar angle on $S^{2}$ in section III. According to separation of variables we write [4]:

$$
\psi(r, \vartheta, t)=R(r) \Theta(\vartheta) T(t)
$$

Substituting this into (A1) we obtain:

$$
\begin{gathered}
R(r) \Theta(\vartheta) \frac{d^{2} T(t)}{d t^{2}}= \\
c^{2}\left[\frac{d^{2} R(r)}{d r^{2}} \Theta(\vartheta) \frac{d^{2} T(t)}{d t^{2}}+\frac{1}{r} \frac{d R(r)}{d r} \Theta(\vartheta) T(t)+\frac{1}{r^{2}} R(r) \frac{d^{2} \Theta(\vartheta)}{d \vartheta^{2}} T(t)\right] .
\end{gathered}
$$

Dividing both sides of this equation by $\mathrm{T}(\mathrm{t})$ we get

$$
\frac{T^{\prime \prime}(t)}{T(t)}=c^{2}\left[\frac{R^{\prime \prime}(r)}{R(r)}+\frac{1}{r} \frac{R^{\prime}(r)}{R(r)}+\frac{1}{r^{2}} \frac{\Theta^{\prime \prime}(\vartheta)}{\Theta(\vartheta)}\right] .
$$

Now in order for equation (A4) to be always true we must have

$$
\frac{T^{\prime \prime}(t)}{T(t)}=-\omega^{2}
$$

with $\omega$ real. The most general complex solution of this
equation having unit magnitude is

$$
T(t)=e^{\imath \omega t}=\cos (\omega t)+\imath \sin (\omega t) .
$$

Looking at the other side of equation (A4) we get

$$
-\omega^{2}=c^{2}\left[\frac{R^{\prime \prime}(r)}{R(r)}+\frac{1}{r} \frac{R^{\prime}(r)}{R(r)}+\frac{1}{r^{2}} \frac{\Theta^{\prime \prime}(\vartheta)}{\Theta(\vartheta)}\right],
$$

or

$$
\frac{-\omega^{2} r^{2}}{c^{2}}=\frac{r^{2} R^{\prime \prime}(r)+r R^{\prime}(r)}{R(r)}+\frac{\Theta^{\prime \prime}(\vartheta)}{\Theta(\vartheta)} .
$$

We can rewrite this equation as

$$
\frac{r^{2} R^{\prime \prime}(r)+r R^{\prime}(r)}{R(r)}+\frac{\omega^{2} r^{2}}{c^{2}}=\frac{-\Theta^{\prime \prime}(\vartheta)}{\Theta(\vartheta)}=\frac{\lambda}{c^{2}}
$$


Just as for equation (A4), we see that in order for this equation to always be true we must insist that

$$
\frac{r^{2} R^{\prime \prime}(r)+r R^{\prime}(r)}{R(r)}+\frac{\omega^{2} r^{2}}{c^{2}}=\frac{-\Theta(\vartheta)}{\Theta(\vartheta)}=\frac{\lambda}{c^{2}}=n^{2} .
$$

Since $\vartheta$ ranges over a compact set, $\vartheta \in[0,2 \pi]$, it follows that $n$ must be an integer. We thus have

$$
\Theta^{\prime \prime}(\vartheta)+\frac{\lambda}{c^{2}} \Theta(\vartheta)=0
$$

The solution of this differential equation is the same as equation (A6),

$$
\Theta(\vartheta)=e^{\imath \eta \vartheta}
$$

From the other side of equation (A9) we obtain

$$
\frac{r^{2} R^{\prime \prime}(r)+r R^{\prime}(r)}{R(r)}=\left(n^{2}-\frac{\omega^{2} r^{2}}{c^{2}}\right),
$$

which we rewrite as

$$
r^{2} R^{\prime \prime}(r)+r R^{\prime}(r)-\left(n^{2}-\frac{\omega^{2} r^{2}}{c^{2}}\right) R(r)=0 .
$$

Now let us show that this can be transformed into Bessel's differential equation, which is [1]

$$
x^{2} y^{\prime \prime}+x y^{\prime}+\left(x^{2}-n^{2}\right) y=0 \text {. }
$$

We let

$$
x=\frac{\omega r}{c} \Rightarrow x^{2}=\frac{\omega^{2} r^{2}}{c^{2}}
$$

and $\frac{d x}{d r}=\frac{\omega}{c}$. Also, $\frac{d}{d r}=\frac{d x}{d r} \frac{d}{d x}=\frac{\omega}{c} \frac{d}{d x}$. Thus,

$$
\frac{r^{2} \omega^{2}}{c^{2}} \frac{d^{2} R}{d x^{2}}+\frac{r \omega}{c} \frac{d R}{d x}+\left(x^{2}-n^{2}\right) R(r)=0
$$

or, since $x=\frac{\omega r}{c}$, we have

$$
x^{2} \frac{d^{2} R(r)}{d x^{2}}+x \frac{d R}{d x}+\left(x^{2}-n^{2}\right) R(r)=0
$$

Now we define $\widetilde{R}(x)=R(r)$ to get

$$
x^{2} \tilde{R}^{\prime}(x)+x R^{\prime}(x)+\left(x^{2}-n^{2}\right) \tilde{R}(x)=0,
$$

which by comparing with equation (A14) we obtain $\quad \tilde{R}(x)=J_{n}(x) \quad$ or $R(r)=J_{n}(\omega r / c)$ where $J_{n}(x)$ is the Bessel function of integer order $n$. 
Summarizing, we have shown that

$$
\psi(r, \vartheta, t)=\widetilde{R}(x) \Theta(\vartheta) T(t)=J_{n}(r \omega / c) e^{i n \vartheta} e^{\imath \omega t}
$$

where $n$ is any positive integer [9] and $\omega$ is any real number which, in order to satisfy the boundary condition $\psi(a, \vartheta, t)=0$, is determined by $J_{n}(a \omega / c)=0$, i.e., $a \omega / c$ is a root of $J_{n}(x)=0$.

\section{REFERENCES}

1. Richard Courant and David Hilbert. Methods of Mathematical Physics, Vol. 1 (Wiley-Interscience, New York, 1989).

2. I.E. Segal and Z. Zhou, Ann. Phys., vol. 218 (1992) pp 279-292.

3. P. Moylan, "The Casimir Effect in the Einstein Universe" [in preparation].

4. Willard Miller Jr. Symmetry and Separation of Variables (Academic Press, New York, 1979).

5. H. Jakobsen and $M$. Vergne, Jour. Funct. Anal., vol. 47 (1982) pp 78-142.

6. Jiri Blank, Pavel Exner and Miloslav Havliček, Hilbert Space Operators in Quantum Physics (AIP Press, New York, 1994).

7. ibid, p 170.
N. Vilenkin, Special Functions and the Theory of Group Representationsrevised edition. (American Mathematical Society, Providence, Rhode Island, USA, 1983).

9. P. Moylan, "Harmonic Analysis on Spannors," Jour. Math. Phys., vol. 36, no. 6 (1995) pp 2826-2879.

10. P. Moylan, "The $\mathrm{SO}(4,4)$ Minimal Representation and the Rac Representation of $\mathrm{SO}(2,3)$ " in Quantum Theory and Symmetries, eds. H.D. Doebner et al. (World Scientific, Singapore, 2000) pp 592596.

11. I.E. Segal and S.M. Paneitz, Jour. Func. Anal., vol. 47 (1982) pp 78-142.

12. I.C. Gradshteyn and I.M. Rhysik, Tables of integrals, Sums, Series and Derivatives (Academic Press, New York, 2000).

13. Michael E. Taylor, Noncommutative Harmonic Analysis (American Mathematical Society, Rhode Island, USA, 1980) p 130.

14. Robert S. Strichartz, Jour. Funct. Anal., vol. 12, no. 4 (1973) pp 341-383.

8.
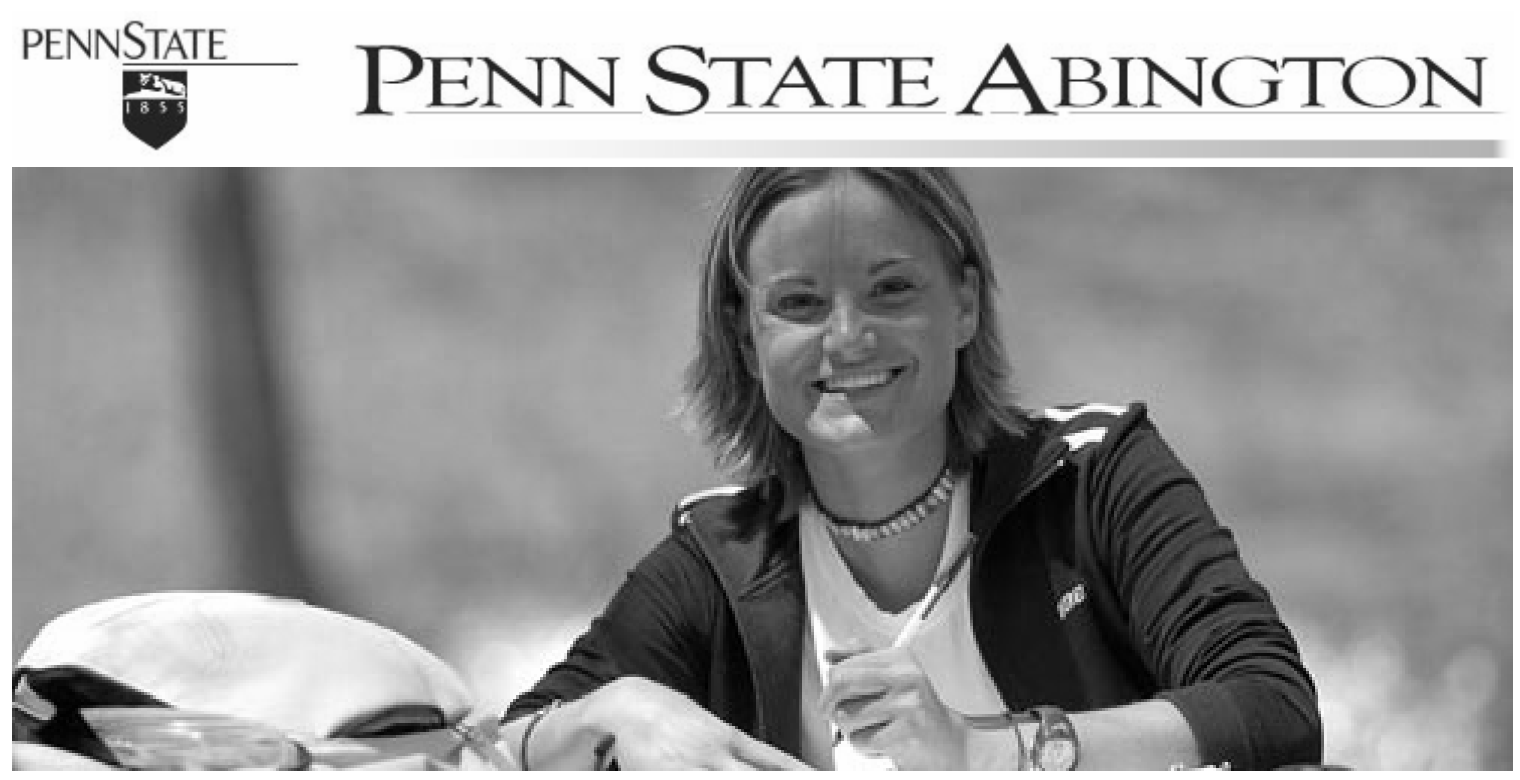

http://www.abington.psu.edu/psasite.php 


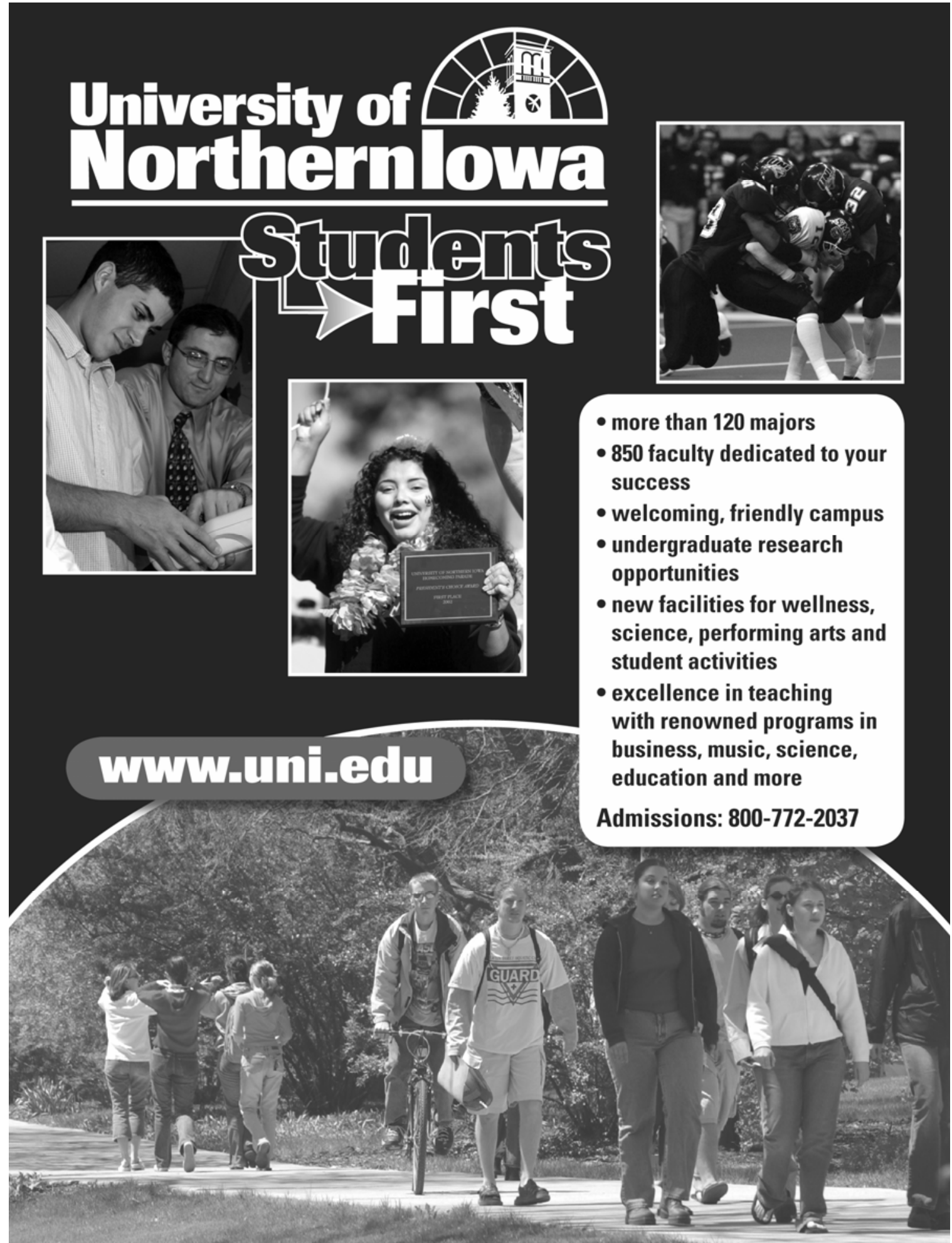

